Auch zur Entkräftung des zwischenzeitlich ergangenen Vorwurfs doppelter Standards bei der Bewertung von Wahlen ihrer Teilnehmerstaaten konzentrieren sich die Beobachtungsaktivitäten der OSZE seit einigen Jahren nicht mehr ausschließlich auf die Länder des ehemaligen Warschauer Paktes, die sich in demokratischen Transformationsprozessen befinden, sondern wenden sich zunehmend auch den Wahlgängen in den etablierten Demokratien unter ihren Teilnehmerstaaten zu. Entsprechend erfolgte die Beobachtung der Wahl zum 17. Deutschen Bundestag am 27. September 2009 durch ODIHR nicht vor dem Hintergrund von Zweifeln an der Integrität des Prozesses, wie dies in der medialen Berichterstattung partiell suggeriert wurde, sondern im Einklang mit dieser Weiterentwicklung der bewährten OSZE-Praxis. In Übereinstimmung mit dieser Praxis handelte es sich bei der nach Deutschland entsandten Mission nicht um eine Wahlbeobachtung im klassischen Sinne, zu deren Aufgaben auch die umfassende und systematische Beobachtung der Geschehnisse am Wahltag gehört hätte, sondern um eine Wahlbewertungsmission, deren 15-köpfiges Expertenteam sich insbesondere mit den Rahmenbedingungen des Urnenganges auseinandersetzte.

Selbst wenn die Gesamtbewertung dieser Rahmenbedingungen äußerst positiv ausgefallen ist, benennt der Abschlussbericht der OSZE-Wahlbewertungsmission einzelne rechtliche Festlegungen, die aus internationaler Sicht verbesserungswürdig erscheinen. Die von der OSZE unterbreiteten Vorschläge könnten Eingang in die Überarbeitung der Wahlgesetzgebung finden, die das Bundesverfassungsgericht dem Gesetzgeber bis zum 20. Juni 2011 aufgegeben hat. Sollte sich der Bundestag dazu entschließen, die Hinweise der ODIHR-Experten bei der Novellierung des Bundeswahlgesetzes zu berücksichtigen, würde dies international sicherlich nicht nur als ein deutliches Bekenntnis zum OSZE-System der kollektiven Sicherheit und seiner Bedeutung verstanden werden, sondern der Reputation der OSZE und des ODIHR sowie deren Empfehlungen gerade auch unter anderen Teilnehmerstaaten stärkeren Nachdruck verleihen.

\title{
Die Bundestagswahl 2009 im Spiegel der repräsentativen Wahlstatistik
}

\author{
Eckhard Jesse
}

Die SPD erreichte bei der Bundestagswahl 2009 in der Gruppe der 25- bis 34-jährigen Männer im Osten Deutschlands bloß 12,8 Prozent, lag damit nicht nur hinter der CDU (25,0 Prozent) und der Linken (19,7 Prozent), sondern auch hinter der FDP (17,8 Prozent); und bei den 18- bis 24-Jährigen - gleiches Geschlecht, gleiche Region - schnitten die „übrigen Parteien“ (20,8 Prozent), darunter die Piratenpartei ${ }^{1}$ und die NPD (8,5 Prozent),

1 Das Statistische Bundesamt hatte die „Piraten“ zunächst nicht berücksichtigt, sie später jedoch einbeziehen wollen. Dies war nur für sieben Länder möglich. Wie die Angaben für Brandenburg (14,0 Prozent), Berlin-Ost (16,4 Prozent) und Mecklenburg-Vorpommern (14,2 Prozent) verdeutlichen, kam die Partei bei den jungen Männern im Osten auf bessere Ergebnisse als die NPD. Wären die „Piraten“ auch in Sachsen angetreten, dann hätten die „übrigen Parteien“ in dieser Kategorie sogar an der ersten Stelle gelegen. 
besser ab als die Linke (19,0 Prozent), FDP (15,7 Prozent), SPD (14,3 Prozent) sowie Grüne (8,5 Prozent) und fast so gut wie die CDU (21,7 Prozent). Bundesweit hatten sie lediglich oder immerhin, je nach Perspektive, 6,0 Prozent erreicht.

Die Länderebene fördert weitere zum Teil höchst überraschende Erkenntnisse zutage. So rangieren die Grünen im Bundesland Berlin in den drei Altersgruppen bis 44 Jahre an erster Stelle. Das beste Ergebnis erzielte die Linke in Sachsen-Anhalt bei den über 45- bis 59-jährigen Männern mit 40,5 Prozent. Diese Partei erreichte im Saarland bei den 45- bis 59-jährigen Männern 28,8 Prozent und nahm damit den Spitzenplatz ein (landesweit bei den Männern: 23,9 Prozent). Bei der ersten gesamtdeutschen Bundestagswahl am 2. Dezember 1990 hatte sie dort 0,2 Prozent erhalten (1994: 0,7 Prozent; 1998: 1,0 Prozent; 2002: 1,4 Prozent; 2005: 18,5 Prozent). Nie lagen die beiden nicht mehr so "Großen“ (56,8 Prozent) derart dicht mit den drei nun nicht mehr ganz „Kleinen“ (37,2 Prozent) zusammen. Daraus resultieren so knappe Ergebnisse wie niemals zuvor in den Untergruppen.

\section{Die Exaktheit der repräsentativen Wahlstatistik}

Die Genauigkeit dieser Zahlen fußt auf der amtlichen repräsentativen Wahlstatistik, die schon seit 1953 besteht und weltweit einzigartig ist. ${ }^{2}$ Der Bundestag setzte sie für die Bundestagswahlen 1994 wie 1998 unverständlicherweise aus: Die als Begründung angeführten datenschutzrechtlichen Bedenken erwiesen sich jedoch nicht als stichhaltig. Bildete zuvor $\$ 51$ des Bundeswahlgesetzes die Rechtsgrundlage ${ }^{3}$, so wurde 1999 ein „Wahlstatistikgesetz" erlassen. ${ }^{4}$ Die Veröffentlichung der Statistik durch den Bundeswahlleiter ${ }^{5}$ mehr als vier Monate nach der Bundestagswahl findet allerdings kaum öffentliches Interesse. Noch später, meist ein Jahr nach der Bundestagswahl, erfolgt die (sparsame) Kommentierung der Daten. ${ }^{6}$ Dabei basiert sie auf der tatsächlichen Stimmabgabe, nicht auf der bekundeten Meinung der Bürger wie bei den Wahltagsbefragungen der demoskopischen Institute.

Allerdings hat Infratest dimap diesmal ausgezeichnete Ergebnisse vorzuweisen. ${ }^{7} \mathrm{Die} \mathrm{Ab}-$ weichungen zur repräsentativen Wahlstatistik sind wahrlich nicht groß: So wurden die Ge-

2 Die Ergebnisse der repräsentativen Wahlstatistik wurden in dieser Zeitschrift dokumentiert und kommentiert. Vgl. Eckhard Jesse, Die Bundestagswahlen von 1953 bis 1972 im Spiegel der repräsentativen Wahlstatistik. Zur Bedeutung eines Schlüsselinstrumentes der Wahlforschung, in: ZParl, 6. Jg. (1975), H. 3, S. 310 - 322; ders., Die Bundestagswahlen von 1972 bis 1987 im Spiegel der repräsentativen Wahlstatistik, in: ZParl, 18. Jg. (1987), H. 2, S. 232 - 242; Hans Rattinger, Das Wahlverhalten bei der ersten gesamtdeutschen Bundestagswahl nach Alter und Geschlecht: Ergebnisse der repräsentativen Wahlstatistik, in: ZParl, 23. Jg. (1992), H. 2, S. 266 - 280; Eckhard Jesse, Die Bundestagswahlen von 1990 und 2002 im Spiegel der repräsentativen Wahlstatistik, in: ZParl, 34. Jg. (2003), H. 4, S. 645 - 656; ders., Die Bundestagswahl 2005 im Spiegel der repräsentativen Wahlstatistik, in: ZParl, 37. Jg. (2006), H. 3, S. 513 - 523.

3 Vgl. für Einzelheiten Wolfgang Schreiber, Kommentar zum Bundeswahlgesetz, Köln 2009, S. 79 f., S. $814-825$.

4 Es ist abgedruckt ebenda, S. $972-974$.

5 Vgl. Statistisches Bundesamt (Hrsg.), Wahl zum 17. Deutschen Bundestag am 27. September 2009, Heft 4: Wahlbeteiligung und Stimmabgabe der Männer und Frauen nach Altersgruppen, Wiesbaden 2010.

6 Vgl. Statistisches Bundesamt (Hrsg.), Wahl zum 16. Deutschen Bundestag am 18. September 2005, Heft 5: Textliche Auswertung der Wahlergebnisse, Wiesbaden 2006.

7 Vgl. Infratest dimap, Wahlreport. Bundestagswahl 27. September 2009, Berlin 2009, S. 55. 
winne der CDU bei den Frauen mit 36 Prozent (richtig: 36,4 Prozent) und die Verluste mit 32 Prozent (richtig: 31,0 Prozent) ebenso gut ermittelt wie die Abstürze der SPD bei den 18- bis 24-jährigen Wählern mit 18 Prozent (richtig: 18,2 Prozent). Wer jedoch Geschlecht und Altersgruppen kombiniert, findet durchaus Abweichungen: Die CDU erreichte bei den über 60-jährigen Männern nicht 41, sondern 39,1 Prozent. Insgesamt kann sich das Ergebnis aber sehen lassen. Das gilt auch für das schwer zu ermittelnde Stimmensplitting. So hieß es, 72 Prozent der Erststimmenwähler der SPD hätten diese auch mit ihrer Zweitstimme gewählt (richtig: 71,0 Prozent) und 13 Prozent die Grünen (richtig: 12,8 Prozent). ${ }^{8}$

In 2861 (2523 allgemeinen und 338 Briefwahlbezirken) von rund 90.000 Wahlbezirken (3,9 Prozent) wurde die Wahlbeteiligung (nach dem Geschlecht und nach zehn Altersgruppen) ausgewertet, ebenso die Stimmabgabe (nach dem Geschlecht und nach fünf Altersgruppen). ${ }^{9}$ Die Stimmzettel in den besagten Wahlbezirken enthielten entsprechende Unterscheidungsaufdrucke, ohne deswegen das Wahlgeheimnis zu verletzen. Wenngleich Geschlecht und Alter das Wahlverhalten nicht vorrangig prägen, lassen die exakten Angaben aufschlussreiche Erkenntnisse zu (die Beteiligungsquote fällt allerdings um 0,6 Punkte überhöht aus, da sämtliche Wahlscheininhaber als Briefwähler zählen, obwohl „nur“ gut 90 Prozent tatsächlich von der Briefwahl Gebrauch machen). Seit 2002 sind auch die Stimmen der Briefwähler einbezogen - durch das Erste Gesetz zur Änderung des Wahlstatistikgesetzes. Angesichts der stetig gestiegenen Briefwahlquote (2009: 21,4 Prozent) war dies unvermeidlich. So gibt es zwischen den Ergebnissen bei der Wahl und den Stichproben der repräsentativen Wahlstatistik keinerlei Abweichungen mehr. Die repräsentative Wahlstatistik ist damit eine wahre Fundgrube für Wahlforscher.

\section{Wablbeteiligung}

Die Zahl der Wähler ist auf einen Tiefstand gesunken, der Anteil der Nichtwähler stellt damit zum ersten Mal in der Geschichte der Bundesrepublik die größte Gruppe. Wie Tabelle 1 zeigt, geht der Rückgang überproportional auf die ohnehin weniger wahleifrigen jüngeren Altersgruppen zurück. Die Partizipationsrate der 60- bis 69-Jährigen sank um 5,0 Punkte, die der über 70-Jährigen um 3,9 Punkte, die der 25- bis 29-Jährigen hingegen um 8,9 Punkte, wobei die Differenz prozentual noch höher ist. Die Wahlbeteiligungskurve weist bei den Bundestagswahlen einen typischen Verlauf auf: beständig steigend von der zweiten Altersgruppe (21 bis 24 Jahre: 59,1 Prozent) bis zur vorletzten (60 bis 69 Jahre: 80,0 Prozent), dann stark fallend, diesmal auf 72,8 Prozent. Die Wahlbereitschaft der 18- bis 20-Jährigen (63,0 Prozent) liegt stets über jener der 21- bis 24-Jährigen (59,1 Prozent). Das hängt nicht mit dem Reiz des Neuen zusammen, sondern mit der weniger starken Familienbindung und der höheren geographischen Mobilität der nächsten Altersgruppe. Die Gründe für den nachlassenden Wahleifer bei den Alten sind in der stärkeren gesellschaftlichen Isolation zu suchen, vor allem im politischen Desinteresse der vielen alleinstehenden Frauen. Die Extremwerte nach Ländern lauten: 45,3 Prozent (die 25- bis 29-jährigen Männer Sachsen-Anhalts) und - fast doppelt so hoch - 84,9 Prozent (die 60- bis 69-jährigen Männer im Saarland).

8 Vgl. ebenda, S. 62.

9 Vgl. für Einzelheiten Statistisches Bundesamt (Hrsg.), a.a.O. (Fn. 5), S. $94-98$. 


\begin{tabular}{|c|c|c|c|c|c|c|}
\hline \multicolumn{7}{|c|}{$\begin{array}{l}\text { Tabelle 1: Wablbeteiligung der Männer und Frauen nach Altersgruppen bei der Bundestagswahl } \\
2009 \text { im Vergleich zur Bundestagswahl } 2005 \text { (Angaben in Prozent; Differenz } \\
\text { in Prozentpunkten)* }\end{array}$} \\
\hline Alter & \multicolumn{2}{|c|}{ Männer } & \multicolumn{2}{|c|}{ Frauen } & \multicolumn{2}{|c|}{ zusammen } \\
\hline 18-20 Jahre & 63,2 & $(-7,1)$ & 62,7 & $(-6,9)$ & 63,0 & $(-7,0)$ \\
\hline 21-24 Jahre & 59,4 & $(-6,7)$ & 58,9 & $(-8,0)$ & 59,1 & $(-7,4)$ \\
\hline 25-29 Jahre & 60,3 & $(-8,7)$ & 62,0 & $(-9,2)$ & 61,2 & $(-8,9)$ \\
\hline 30-34 Jahre & 64,2 & $(-8,9)$ & 65,9 & $(-10,0)$ & 65,1 & $(-9,4)$ \\
\hline 35-39 Jahre & 68,0 & $(-9,0)$ & 69,9 & $(-10,0)$ & 68,9 & $(-9,5)$ \\
\hline 40-44 Jahre & 71,6 & $(-7,2)$ & 73,2 & $(-7,4)$ & 72,4 & $(-7,5)$ \\
\hline 45-49 Jahre & 72,6 & $(-6,5)$ & 73,8 & $(-7,5)$ & 73,2 & $(-7,0)$ \\
\hline 50-59 Jahre & 74,3 & $(-7,6)$ & 75,2 & $(-7,9)$ & 74,8 & $(-7,7)$ \\
\hline 60-69 Jahre & 80,4 & $(-5,1)$ & 79,7 & $(-4,9)$ & 80,0 & $(-5,0)$ \\
\hline ab 70 Jahre & 78,4 & $(-4,3)$ & 69,1 & $(-4,0)$ & 72,8 & $(-3,9)$ \\
\hline alle & 71,8 & $(-6,7)$ & 71,0 & $(-7,1)$ & 71,4 & $(-6,9)$ \\
\hline \multicolumn{7}{|c|}{$\begin{array}{l}\text { * Anteil der Wähler mit Stimm- und Wahlscheinvermerk im Wählerverzeichnis an den Wahlberechtigten. } \\
\text { Lesehinweis: Die Wahlbeteiligung der 40- bis 44-jährigen Männer betrug } 2009 \text { 71,6 Prozent und damit } \\
\text { 7,2 Prozentpunkte weniger als } 2005 \text {. } \\
\text { Quelle: Statistisches Bundesamt (Hrsg.), a.a.O. (Fn. 5), S. } 11 \text {. }\end{array}$} \\
\hline
\end{tabular}

Bisher gibt es trotz wichtiger Arbeiten zu den Nichtwählern ${ }^{10}$ keine empirische Studie ${ }^{11}$, die den Gründen für die Konstanz der Wahlbeteiligungskurve nachgeht. ${ }^{12}$

Die Beteiligungsrate der Frauen ist seit einigen Wahlen fast auf dem Niveau der Männer angesiedelt, in den neuen Bundesländern (65,4 zu 64,9 Prozent) sogar stets darüber (in den alten: 72,4 zu 73,5 Prozent), ebenso bei den 25- bis 59-Jährigen. Die „Emanzipation mit dem Stimmzettel“13 ist damit weit fortgeschritten. Der knappe Vorsprung der Männer basiert auf der starken Diskrepanz in der letzten Altersgruppe (Männer: 78,4 Prozent; Frauen: 69,1 Prozent). Gemessen an der durchschnittlichen Wahlbeteiligung von 71,4 Prozent lag sie in den Altersgruppen bis 40 Jahre darunter und in allen Altersgruppen ab 40 Jahren darüber. Dabei gibt es allerdings eine spezifische Ausnahme: Während die über 70-jährigen Männer mit 5,6 Punkten über der Wahlbeteiligungsquote liegen, trifft dies bei den über 70 -jährigen Frauen nicht zu (minus 2,3 Punkte). Das ist ein bekanntes Phänomen aus früheren Bundestagswahlen. Hingegen fällt die Wahlbeteiligung der über 70-Jährigen zum ersten Mal höher aus als die der gesamten Bevölkerung.

10 Vgl. Michael Eilfort, Die Nichtwähler. Wahlenthaltung als Form des Wahlverhaltens, Paderborn 1994; nicht nur bezogen auf die Bundesrepublik Deutschland: Dorothée de Nève, NichtwählerInnen - eine Gefahr für die Demokratie?, Opladen 2009.

11 Vgl. ansatzweise Kai Arzheimer, Jung, dynamisch, Nichtwähler? Der Einfluss von Lebensalter und Kohortenzugehörigkeit auf die Wahlbereitschaft, in: Edeltraud Roller / Frank Brettschneider / Jan W. van Deth (Hrsg.), Jugend und Politik: „Voll normal!“ Der Beitrag der politischen Soziologie zur Jugendforschung, Wiesbaden 2006, S. 317 - 335.

12 So liegt seit 1987 die höchste Wahlbeteiligungsrate stets bei den 60- bis 69-Jährigen, nicht mehr bei den 50 - bis 59-Jährigen. Diese zunehmende Diskrepanz zwischen beiden Altersgruppen 1987: 0,3 Punkte; 1990: 2,1 Punkte; 2002: 2,4 Punkte; 2005: 2,5 Punkte; 2009: 5,2 Punkte - ist klärungsbedürftig.

13 Vgl. Joachim Hofmann-Göttig, Emanzipation mit dem Stimmzettel. 70 Jahre Frauenwahlrecht in Deutschland, Bonn 1986. 
Unter dem Strich gilt: Während die Wahlbeteiligungsrate bei den Geschlechtern immer mehr eingeebnet ist, nimmt das Altersgefälle stärker zu. Beteiligten sich bei den 60- bis 69-Jährigen vier von fünf Wahlberechtigten, so waren es bei den 21- bis 24-Jährigen nicht einmal drei von fünf. Dieser Trend hatte bereits in den achtziger Jahren eingesetzt. „Derartige differentielle Verschiebungen können von beträchtlicher politischer Bedeutung sein, da die Altersgruppen tendenziell unterschiedliche parteipolitische Präferenzen haben." ${ }^{4}$

\section{Stimmabgabe nach dem Geschlecht}

Die Stimmabgabe nach dem Geschlecht ist für Männer und Frauen beim Elektorat der CDU/CSU gegenläufig (vgl. Tabelle 2). Diese verlor bei den Männern 3,8 Punkte (CDU: 2,7; CSU: 1,1), gewann hingegen bei den Frauen 0,9 Punkte (CDU: 1,6; CSU: -0,7). Damit ist die Union (Frauen: 36,4 Prozent; Männer: 31,0 Prozent) - die CDU mehr als die CSU - klar eine „Frauenpartei“ wie in den 50er, 60er und 70er Jahren. Die Gründe dürften unterschiedlicher Natur sein. Seinerzeit repräsentierten diese Parteien ein traditionsverhaftetes Frauenbild, heute verkörpern sie mit Angela Merkel und Ursula von der Leyen Aufgeschlossenheit und Fortschrittlichkeit - so hat das Bemühen um die Frauen Früchte getragen. Ausschließlich 1980 und 2002, unter den beiden Kanzlerkandidaten aus den Reihen der CSU (Franz Josef Strauß und Edmund Stoiber), die nicht für so genannte weiche Themen standen, fiel die Zustimmung der Frauen für die Union hinter die der Männer zurück. Bereits 2005 wurde eine Trendwende eingeleitet: Ihre Einbußen bei den Frauen (2,3 Punkte) waren seinerzeit nur etwa halb so hoch wie bei den Männern (4,4 Punkte).

Die SPD hingegen verlor 2009 bei den Frauen 2,6 Punkte mehr als bei den Männern. Diese Tendenz gab es - wenngleich deutlich schwächer - schon 2005. Minimal wählten Frauen (23,1 Prozent) sie 2009 häufiger als Männer (23,0 Prozent), anders als in den 50er, 60er und 70er Jahren. Bei der Wählerschaft der drei kleineren Parteien traten geschlechtsspezifische Eigentümlichkeiten verstärkt hervor. Die Liberalen (16,1:13,1 Prozent) und vor allem die Postkommunisten (13,3:10,6 Prozent) sind immer mehr eine „Männerpartei“, die Grünen (12,0:9,4 Prozent) eine „Frauenpartei“ geworden. Deren Wandel ist frappierend. Die Überrepräsentanz bei den Frauen seit den 90er Jahren nimmt von Wahl zu Wahl zu. In ihren Anfängen waren die Grünen eine Männerpartei. ${ }^{15}$ Die Dominanz der Männer bei den „Sonstigen“ (7,3:4,8 Prozent) resultiert zur Hälfte aus der Wahl der rechtsextremistischen NPD, die wie jede radikale Kraft notorisch mehr Männer als Frauen anzieht (2,1:0,8 Prozent). In der Forschung gibt es keinen Konsens bei der Frage nach einem geschlechtsspezifischen Wahlverhalten. ${ }^{16}$

14 So Jürgen W. Falter / Siegfried Schumann, Der Nichtwähler - das unbekannte Wesen, in: HansDieter Klingemann / Max Kaase (Hrsg.), Wahlen und Wähler. Analysen aus Anlass der Bundestagswahl 1990, Opladen 1994, S. 161 - 213, S. 207.

15 So erzielten die Grünen 1983 bei den Männern 5,9 Prozent (1987: 8,3), bei den Frauen nur 4,8 Prozent $(1987:$ 7,7).

16 Vgl. Jürgen W. Falter / Siegfried Schumann, Vive la très petite difference. Über das unterschiedliche Wahlverhalten von Männern und Frauen bei der Bundestagswahl 1987, in: Max Kaase I Hans-Dieter Klingemann (Hrsg.), Wahlen und Wähler. Analysen aus Anlass der Bundestagswahl 1987, Opladen 1990, S. $109-142$. 


\begin{tabular}{|c|c|c|c|c|c|c|}
\hline Partei & & & & & & nen \\
\hline CDU/CSU & 31,0 & $(-3,8)$ & 36,4 & $(+0,9)$ & 33,8 & $(-1,4)$ \\
\hline SPD & 23,0 & $(-9,8)$ & 23,1 & $(-12,4)$ & 23,0 & $(-11,2)$ \\
\hline FDP & 16,1 & $(+5,4)$ & 13,1 & $(+4,1)$ & 14,6 & $(+4,8)$ \\
\hline Die Linke & 13,3 & $(+3,4)$ & 10,6 & $(+3,0)$ & 11,9 & $(+3,2)$ \\
\hline B 90/Grüne & 9,4 & $(+2,0)$ & 12,0 & $(+3,2)$ & 10,7 & $(+2,6)$ \\
\hline Sonstige & 7,3 & $(+2,9)$ & 4,8 & $(+1,3)$ & 6,0 & $(+2,0)$ \\
\hline
\end{tabular}

\section{Stimmabgabe nach dem Alter}

Die Stimmabgabe nach Altersgruppen verzeichnet deutlichere Unterschiede als die nach dem Geschlecht - nicht nur bei dieser Wahl. ${ }^{17}$ Union, SPD (nicht in der Vergangenheit) - mit dem stärksten Anteil jeweils in der Kategorie der über 60-Jährigen - und die Linke ${ }^{18}$ sind eher „alte" Parteien, Grüne und Liberale (vor 2002 war das wechselnd) ,junge“ (vgl. Tabelle 3). Jeweils eine Altersgruppe fällt aus dieser Systematik heraus: Bei der CDU/CSU ist es die der 45- bis 59-Jährigen, bei der SPD die der 25- bis 34-Jährigen, bei der FDP die der 18- bis 24-Jährigen, bei der Linken die Gruppe der über 60-Jährigen und bei den Grünen die der 35- bis 44-Jährigen. Krass ist die Differenz bei den Grünen: Die 18- bis 24-Jährigen votierten dreimal mehr als die 60-Jährigen für sie. Die These vom „Ergrauen der Grünen "19 ist damit nur bedingt richtig.

Mehrere Verschiebungen gegenüber der letzten Wahl fallen auf: Die zwei größeren Parteien haben in allen fünf Alterskategorien Stimmen eingebüßt, die drei kleineren gewonnen. Während der Anteil der Union bei den 18- bis 24-Jährigen fast unverändert geblieben ist, brach die SPD, für Jungwähler unattraktiv geworden, hier mit einem Verlust von 18,7 Punkten geradezu ein (jetzt: 18,2 Prozent). Ohne die gesunkene Wahlbeteiligung, die die Verluste noch stärker verdeutlichen, zu berücksichtigen: Die SPD hat in dieser Altersgruppe mehr als jede zweite Stimme verloren und in der nächsten fast jede zweite. Zum ersten Mal überflügelte die FDP in einer Altersgruppe die SPD (bei den 25- bis 34-Jährigen). Deren Verluste sinken mit dem höheren Alter der Wählerschaft. Sie betragen bei den über

17 Vgl. etwa Jürgen W. Falter / Uwe W. Gehring, Alter - ein neues Cleavage?, in: Max Kaase / HansDieter Klingemann (Hrsg.), Wahlen und Wähler. Analysen aus Anlass der Bundestagswahl 1994, Opladen 1998, S. $463-503$.

18 Allerdings sind die Unterschiede bei der Linken nicht so groß wie bei der Union und der SPD.

19 Vgl. Wilhelm P. Bürklin / Russell J. Dalton, Das Ergrauen der Grünen, in: Hans-Dieter Klingemann / Max Kaase (Hrsg.), a.a.O. (Fn. 14), S. 264 - 302; Markus Klein / Kai Arzheimer, Grau in Grau. Die Grünen und ihre Wähler nach eineinhalb Jahrzehnten, in: Kölner Zeitschrift für Soziologie und Sozialpsychologie, 49. Jg. (1997), H. 4, S. 650 - 673; Ulrich Kohler, Zur Attraktivität der Grünen bei älteren Wählern, in: Kölner Zeitschrift für Soziologie und Sozialpsychologie, 50. Jg. (1998), H. 3, S. $536-559$. 
60-Jährigen lediglich ein gutes Drittel der ersten Altersgruppe. Dürften die erneut überproportional hohen Gewinne der Linken bei den 45- bis 59-Jährigen (mit einer Steigerung von 4,1 Punkten gegenüber 2005 und 10,5 Punkten gegenüber 2002) wesentlich der Existenzangst von Arbeitnehmern und Arbeitslosen geschuldet sein, so ist das vergleichsweise schwache Ergebnis (nicht im Osten bei den Männern) in der Gruppe der über 60-Jährigen unter anderem gerade mit dem Fehlen dieser Existenzangst erklärbar. Das überaus gute Resultat der „Sonstigen“ in der Kategorie „18 bis 24 Jahre“ wurzelt vor allem in der Wählerstruktur der Piratenpartei und der NPD.

Zwei Punkte verdienen es hervorgehoben zu werden. Zum einen kann die Aufgliederung zum Missverständnis verleiten, die fünf Altersgruppen seien ungefähr gleich stark. Tatsächlich ist das Größenverhältnis - der Wähler, nicht der Wahlberechtigten - folgendermaßen: 8,0 Prozent, 11,7 Prozent, 17,0 Prozent, 28,6 Prozent, 34,7 Prozent. Damit umfassen die über 60-Jährigen fast so viele Wähler wie die der ersten drei Kategorien zusammen. Die Parteien wussten, so darf vermutet werden, warum sie vor den Wahlen eine großzügige Rentenerhöhung verabschiedeten. Zum anderen verstellt die Fixierung auf die Alten eine wichtige Einsicht. Es gibt nicht nur ein altersspezifisches Wahlverhalten, wie die George Bernard Shaw zugeschriebenen Worte nahe legen („Wer mit 20 nicht links ist, hat kein Gefühl, wer es mit 40 noch ist, keinen Verstand"), sondern auch ein generationenspezifisches. So können bestimmte Ereignisse das Weltbild einer ganzen Kohorte prägen. Der Rückgang der Stammwähler relativiert beide Thesen.

\section{Kombination von Alter und Geschlecht}

Wer Geschlecht und Altersgruppen kombiniert, erfährt Details, die bisher unbeachtet blieben (vgl. Tabelle 4). Die Union hat - wie 2005 - ihr schwächstes Ergebnis bei den männlichen 18- bis 24-jährigen Wählern (24,0 Prozent) erreicht, ihr bestes bei den über 60-jährigen Frauen (45,3 Prozent). Die SPD erzielte ihr schlechtestes Resultat bei den männlichen 25- bis 34-jährigen Wählern (16,1 Prozent), ihr bestes bei den über 60-jährigen Männern (27,6 Prozent). 2005 hingegen war dies für die Partei die zweitschlechteste der zehn Kategorien. Die SPD-Wählerschaft ist damit älter und „männlicher“ geworden, die der Union jünger und „weiblicher“. Die FDP vermochte in der Gruppe der männlichen 25- bis 34-Jährigen 20,5 Prozent auf sich zu vereinigen, die Linke 16,9 Prozent bei den männlichen 45- bis 59-jährigen Wählern. Geradezu sensationell ist das Ergebnis für die Grünen bei den 18- bis 24-jährigen Frauen: 18,9 Prozent. Hingegen wären die Grünen mit ihren 4,6 Prozent bei den männlichen Wählern über 60 Jahre an der Fünfprozenthürde gescheitert. Die FDP und die Linke hatten ebenso ihre schwächsten Resultate in der letzten Alterskategorie, aber bei den Frauen. Wenn die „sonstigen Parteien“ unter den 18- bis 24-jährigen Männern 18,9 Prozent erreichen und „bloß“ 10,2 Prozent bei den gleichaltrigen Frauen, so geht dies nicht nur auf die NPD zurück, sondern auch auf die Piratenpartei, die einen hohen "gender gap“ aufweist - am stärksten in Hessen (13,0:3,9 Prozent), am schwächsten in Brandenburg (14,0:6,0 Prozent).

Hinter den Verlusten der Union von 0,3 Punkten bei den 18- bis 24-Jährigen verbergen sich Gewinne (1,7 Punkte) bei den Frauen und Einbußen (2,3 Punkte) bei den Männern; und ein zweiter Blick auf die Gewinne bei den weiblichen 18- bis 24-jährigen Wählern zeigt gegenläufige Ergebnisse der Unionsparteien (nicht bei den Verlusten der männlichen 


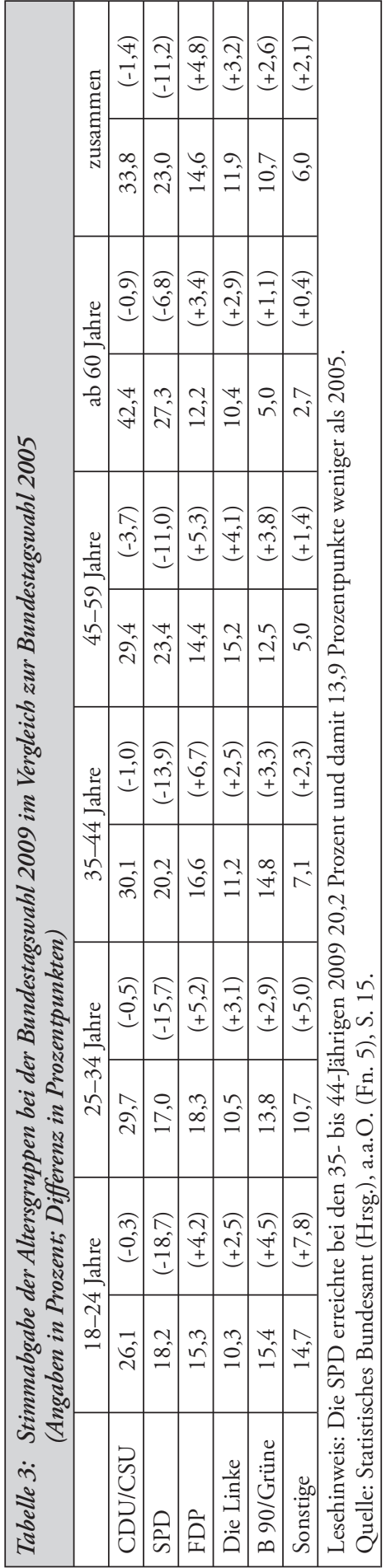

\begin{tabular}{|c|c|c|c|c|c|c|c|c|c|}
\hline & 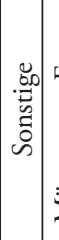 & 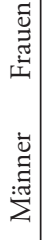 & 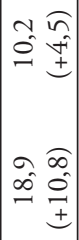 & 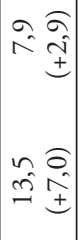 & 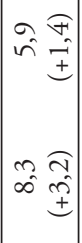 & 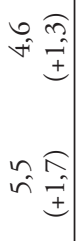 & 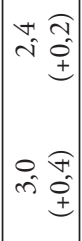 & 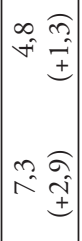 & \\
\hline 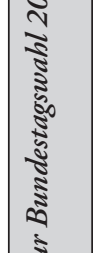 & 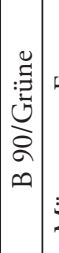 & 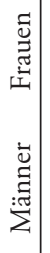 & 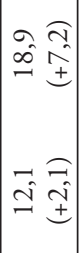 & 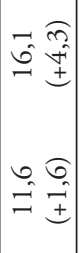 & 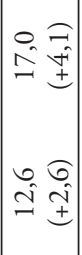 & 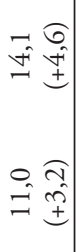 & 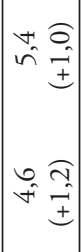 & 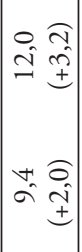 & 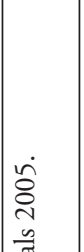 \\
\hline 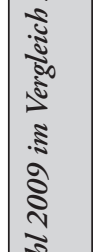 & 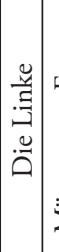 & 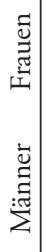 & 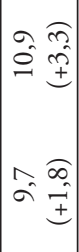 & 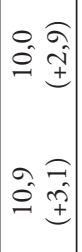 & 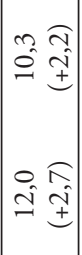 & 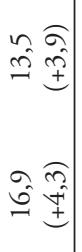 & 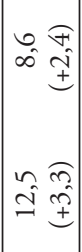 & 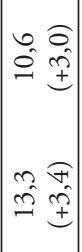 & 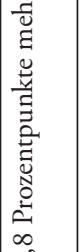 \\
\hline 5 & 灾 & 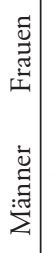 & 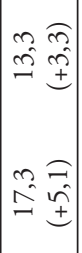 & 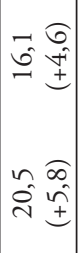 & 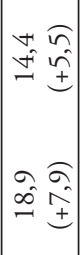 & 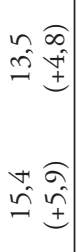 & 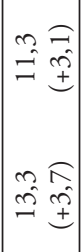 & 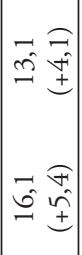 & 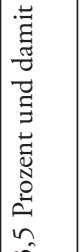 \\
\hline 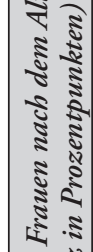 & $\overrightarrow{\hat{\omega}}$ & 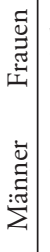 & 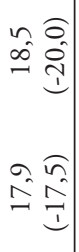 & 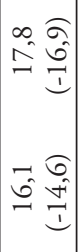 & 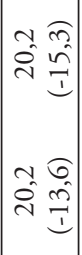 & 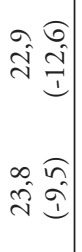 & 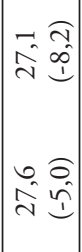 & 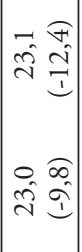 & 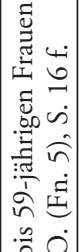 \\
\hline 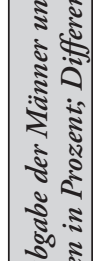 & $\begin{array}{l}2 \\
\tilde{z} \\
0 \\
2 \\
0 \\
0\end{array}$ & 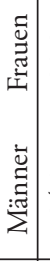 & 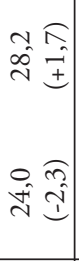 & $\begin{array}{cc}m & = \\
\hat{v} & \vec{?}\end{array}$ & $\left|\begin{array}{cc}\tilde{c} & \widehat{\sigma} \\
\tilde{n} & \overline{ \pm}\end{array}\right|$ & 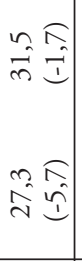 & 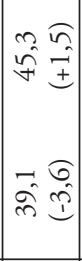 & 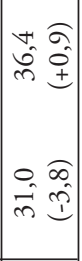 & 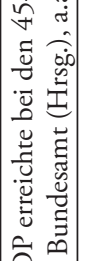 \\
\hline के & 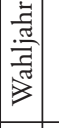 & & 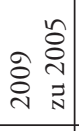 & 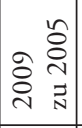 & 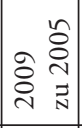 & 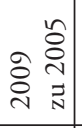 & 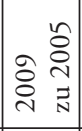 & 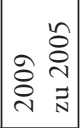 & 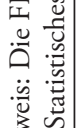 \\
\hline$\frac{5}{8}$ & 节 & & 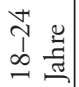 & 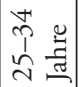 & 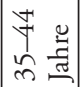 & 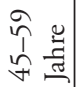 & 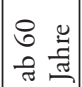 & 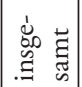 & 记 \\
\hline
\end{tabular}


18- bis 24-Jährigen von 1,1 Punkten bei der CDU und 1,2 Punkten bei der CSU): Während die CDU 2,5 Punkte gewann, verlor die CSU 0,8. Die SPD gab in dieser Altersgruppe bei den Frauen 20,0 Prozentpunkte ab, bei den Männern „nur“ 17,5. Damit ist sie hier schwächer (18,5 Prozent) als die Grünen (18,9 Prozent). Die Liberalen konnten sich bei den 35- bis 44-jährigen Männern um 7,9 Punkte steigern, die Grünen um 7,2 Punkte bei den 18- bis 24-jährigen Frauen. Das sind die beiden größten Zuwächse. Die weiblichen 18- bis 24-Jährigen wählten die „Männerpartei“ Die Linke mit 10,9 Prozent öfter als die männlichen (9,7 Prozent), die hier ihren geringsten Anstieg verbuchen konnte.

\section{Stimmensplitting}

Die repräsentative Wahlstatistik ermöglicht auch genaue Angaben zum Stimmensplitting. Wählten 1957 6,4 Prozent mit ihrer Erststimme eine andere Partei als mit ihrer Zweitstimme, waren es 1990 bereits 15,6 Prozent, zehn Jahre später 20,5 und 2005 23,9 Prozent; 2009 ist dieser Anteil auf 26,4 Prozent gestiegen. Die Gründe sind mannigfach. Der Wähler kann die Erststimme einem aussichtsreichen Kandidaten einer anderen Partei geben oder dem des potentiellen Koalitionspartners. Die Vielzahl und die Größe der im Parlament vertretenen Parteien begünstigt Stimmensplitting ebenso wie die Erosion der herkömmlichen Milieus. Bei der Interpretation dieser Daten ist Vorsicht geboten, unter anderem deswegen, weil nicht jeder Wahlbürger die Zweitstimme als erst- und die Erststimme als zweitrangig ansieht. Die Union erreichte 5,6 Prozentpunkte mehr Erststimmen, die SPD 4,9. Die FDP büßte 7,0 Punkte ein, Bündnis 90/Grüne 1,7 Punkte, die Linke 0,8. Die nahe liegende Annahme, die Linke habe mehr Kernwähler, entspricht so nicht den Tatsachen. Schließlich votierten viele überzeugte FDP-Zweitstimmenwähler bewusst für einen Kandidaten der Union. Insofern ist das ohnehin verräterische Wort von den „Leihstimmen“ verfehlt. Der Einzug der Liberalen in den neuen Bundestag stand selbst für größte Skeptiker niemals in Frage.

Die Zweitstimmenwähler der Union gaben ihre Erststimme zu 87,6 Prozent für den eigenen Kandidaten ab, die der SPD zu 85,6 Prozent (vgl. Tabelle 5). Diese Werte, gegenüber den früheren Wahlen leicht gesunken, belegen die nach wie vor enge Bindung der Wähler der großen Parteien an ihre Kandidaten. Diesmal fielen allerdings 16 Wahlkreise soviel wie nie zuvor - an eine „Drittpartei“, die Linke. Unionswähler stimmen am ehesten - trotz Aussichtslosigkeit - für einen Bewerber der FDP, die der SPD für einen der Grünen. Bei der FDP und den Grünen sind die Abweichungen deutlich größer. Nur 44,8 Prozent der FDP-Zweitstimmenwähler und 53,6 Prozent der Zweitstimmenwähler der Grünen kreuzten einen Kandidaten „ihrer“ Partei an. Die Splitting-Wähler wollten denen einer großen Partei helfen. FDP-Stimmen wanderten vor allem an Bewerber ihres Koalitionspartners, die der Grünen vornehmlich an die der SPD. Die Wähler nahmen die Koalitionssignale der Parteien wahr und handelten dementsprechend. ${ }^{20}$ Die klar engere Bindung der FDP-Wähler an die Union als jene der Grünen-Wähler an die SPD ist erkennbar - erstens im Ausmaß, zweitens in der Richtung des Stimmensplittings. Die Grünen votierten im

20 Vgl. Franz Urban Pappi / Alexander Herzog / Ralf Schmitt, Koalitionssignale und die Kombination von Erst- und Zweitstimmen bei den Bundestagswahlen 1953 bis 2005, in: ZParl, 37. Jg. (2006), H. 3, S. $493-513$. 


\begin{tabular}{|c|c|c|c|c|c|c|c|c|c|c|}
\hline \multirow{3}{*}{$\begin{array}{l}\text { Zweitstimme } \\
\quad=100 \\
\text { CDU/CSU }\end{array}$} & \multicolumn{10}{|c|}{ Erstimmen } \\
\hline & \multicolumn{2}{|c|}{ CDU/CSU } & \multicolumn{2}{|c|}{ SPD } & \multicolumn{2}{|c|}{ FDP } & \multicolumn{2}{|c|}{ Die Linke } & \multicolumn{2}{|c|}{ B 90/Grüne } \\
\hline & 87,6 & $(-3,3)$ & 4,1 & $(+1,2)$ & 4,8 & $(+1,3)$ & 0,7 & $(+0,2)$ & 1,7 & $(+0,8)$ \\
\hline SPD & 4,5 & $(+1,2)$ & 85,8 & $(-0,9)$ & 1,1 & $(-0,1)$ & 2,4 & $(-0,1)$ & 5,2 & $(-0,1)$ \\
\hline FDP & 45,8 & $(-14,4)$ & 4,8 & $(-2,3)$ & 44,8 & $(+15,8)$ & 1,1 & $(-0,3)$ & 2,1 & $(+0,7)$ \\
\hline Die Linke & 3,1 & $(-0,8)$ & 12,8 & $(-4,5)$ & 1,7 & $(+0,2)$ & 75,7 & $(+3,6)$ & 4,8 & $(+2,0)$ \\
\hline B 90/Grüne & 6,1 & $(+1,5)$ & 33,3 & $(-23,4)$ & 2,1 & $(-1,0)$ & 3,6 & $(+1,6)$ & 53,6 & $(+18,9)$ \\
\hline \multicolumn{11}{|c|}{$\begin{array}{l}\text { Lesehinweis: Von den Wählern, die } 2009 \text { mit der Zweitstimme FDP gewählt haben, gaben 4,8 Prozent } \\
\text { ihre Erststimme der SPD und damit 2,3 Punkte weniger als } 2005 \text {. } \\
\text { Quelle: Statistisches Bundesamt (Hrsg.), a.a.O. (Fn. 5), S. 19; Statistisches Bundesamt (Hrsg.), a.a.O. }\end{array}$} \\
\hline
\end{tabular}

Verhältnis von 5,5:1 für Bewerber der SPD (im Vergleich zur Union), die Liberalen im Verhältnis von 9:1 für die der Union (im Vergleich zur SPD). Die Zweitstimmenwähler der Linken legten eine größere Treue zu „ihrer“ Partei an den Tag als die der Liberalen und der Grünen. Nur 24,3 Prozent gaben Bewerbern anderer Parteien die Erststimme - offenkundig deshalb, weil ihre Wähler wissen, dass die eigene Partei für eine Koalition ausscheidet. Zwar votierten sie im Verhältnis von 4:1 für die Kandidaten der SPD im Vergleich zu jenen der Union, doch kann von einem „linken Lager“ nicht die Rede sein, wenn nur 12,8 Prozent mit ihrer Erststimme für Bewerber der Sozialdemokraten votierten. Und die SPDZweitstimmenwähler präferierten lediglich zu 2,4 Prozent die Bewerber der Linken.

Allerdings ist bei der Interpretation des Stimmensplittings Zurückhaltung angebracht. Augenscheinlich versteht ein Teil des Elektorats das Zweistimmensystem nicht. ${ }^{21}$ Dies entschlüsseln die Präferenzen der Erststimmenwähler: Die der FDP wählten zu 17,3 Prozent mit der Zweitstimme die Union, die der Grünen zu 13,1 die SPD. Das ist nicht logisch und kaum mit einer Präferenz für den jeweiligen Wahlkreiskandidaten erklärbar. Schließlich gaben die FDP-Erststimmenwähler bloß zu 2,7 Prozent der SPD ihrer Zweitstimme, die der Grünen zu 6,2 Prozent der Union. 7,3 Prozent aller Wähler blieben der FDP mit beiden Stimmen treu, 19,3 Prozent kreuzten die Partei zumindest einmal an.

\section{Unterschiede und Parallelen zwischen West-und Ostdeutschland}

Das Wahlverhalten in den neuen Ländern unterscheidet sich bekanntlich von dem in den alten - aufgrund sozialisations- (höhere Staatsgläubigkeit) und situationsbedingter (höhere Arbeitslosigkeit) Faktoren. ${ }^{22}$ Es gibt allerdings auch Angleichungstendenzen - mit Blick auf

21 Vgl. Rüdiger Schmitt-Beck, Denn sie wissen nicht, was sie tun ... Zum Verständnis des Verfahrens der Bundestagswahl bei westdeutschen und ostdeutschen Wählern, in: ZParl, 24. Jg. (1993), H. 2, S. $393-415$.

22 Vgl. unter anderem Hans Rattinger / Oscar W. Gabriel / Jürgen W. Falter (Hrsg.), Der gesamtdeutsche Wähler. Stabilität und Wandel des Wählerverhaltens im wiedervereinigten Deutschland, Baden-Baden 2007. 
die Linke und die Union. ${ }^{23}$ Die im Wahlgebiet Ost schwächere Partei gewann hier 4,5 Punkte hinzu, während sie im Wahlgebiet West 2,9 Punkte verlor. Was die Wahlbeteiligung im Osten und im Westen betrifft, findet eine Angleichung nicht statt. Die ohnehin höhere Wahlabstinenz im Osten setzte sich überproportional stark fort: Die Beteiligung sank dort um 9,4 Punkte auf 65,1 Prozent, im Westen um 6,3 Punkte auf 72,9 Prozent.

Wie ein gesonderter Blick auf die alten und neuen Länder (genauer: früheres Bundesgebiet und Berlin-West sowie früheres DDR-Gebiet und Berlin-Ost) erhellt ${ }^{24}$, sind die Unterschiede bei der Linken zwischen Männern und Frauen im Osten eher gering (29,6 zu 27,5 Prozent), im Westen hingegen beträchtlich (9,8 zu 7,0 Prozent). Das ist unter anderem ein Indiz für die größere Radikalität der Partei in den alten Bundesländern. Hier weist sie bei den über 60-Jährigen mit 6,0 Prozent klar unterdurchschnittliche Werte (insgesamt: 8,3 Prozent), in den neuen hingegen mit 30,6 fast ebenso klar überdurchschnittliche (insgesamt: 28,5 Prozent) auf, wobei die Frauen in dieser Alterskategorie die Partei „nur“ zu 27,6 Prozent wählen, die Männer hingegen zu 34,2 Prozent. Waren die Unterschiede bei der Linkspartei 2005 zwischen dem besten Ergebnis (30,8 Prozent bei den 45- bis 59-jährigen Männern im Osten) und dem schwächsten (2,3 Prozent bei den über 60-jährigen Frauen im Westen) eklatant, ist nun eine gewisse, allerdings schwache Annäherung erfolgt: 34,5 Prozent (im Osten bei den 45- bis 59-jährigen Männern) stehen 4,4 Prozent (im Westen bei den über 60-jährigen Frauen) gegenüber. Die Linke gewinnt im für sie schwachen Westen stärker, im für sie starken Osten schwächer. Sie hat damit erstmals mehr Stimmen - in absoluten Zahlen - im Westen als im Osten erreicht.

Während die Union in den alten Bundesländern in jeder Altersgruppe vorne liegt, trifft dies für den Osten bei den 45- bis 59-Jährigen nicht zu (Die Linke: 33,4 Prozent; CDU: 28,3 Prozent). Bei der Union haben sich die Extremwerte etwas eingeebnet. Votierten 2005 18,2 Prozent der 18- bis 24-jährigen Männer im Osten für die CDU, so waren es 47,2 der über 60-jährigen Frauen im Westen. Jetzt lauten die Zahlen: 47,1:21,7 Prozent. Die Wählerschaft der SPD ist im Osten etwas älter als im Westen und dort etwas „weiblicher“ als im Osten, die der FDP im Osten deutlich jünger als im Westen, die der Grünen ein wenig jünger. Insgesamt sind die Unterschiede nicht auffallend (zumal bei der Union).

Die beiden Hauptcharakteristika des Wahlausgangs 2009 - zum einen deutliche Überrepräsentanz der Union bei den Frauen gegenüber 2005, zum anderen massive Verluste der SPD bei den jungen „Wahlbürgern“ - zeigen sich nicht gleichermaßen in den neuen wie den alten Bundesländern. Manche Unterschiede sind mehr als Nuancen. Differieren die SPD-Verluste bei den 18- bis 24-Jährigen im Westen (18,9 Punkte) und im Osten (18,8 Punkte) kaum (insofern ist Ost gleich West) ${ }^{25}$, so sieht es gänzlich anders bei der Wählerschaft für die Union nach dem Geschlecht aus. Hat die Union bei den Männern in den alten Bundesländern 5,2 Punkte verloren und in den neuen 1,6 Punkte gewonnen, so büßte sie bei den Frauen in den alten Bundesländern 0,6 Punkte ein, in den neuen legte sie hingegen 7,3 Punkte zu. Das ist ein sensationelles Ergebnis. Es gibt Anlass, nach den Gründen für die beträchtliche Differenz Ausschau zu halten.

23 Vgl. Eckhard Jesse, Der glanzlose Sieg der „Bürgerlichen“ und die Schwäche der Volksparteien, in: Zeitschrift für Politik, 56. Jg. (2009), H. 4, S. 397 - 408, S. 402.

24 Vgl. Statistisches Bundesamt (Hrsg.), a.a.O. (Fn. 5), S. 70 f.

25 Allerdings fallen die prozentualen Verluste im Westen größer aus. Hier sank die SPD von 37,9 auf 19,0 Prozent, im Osten von 33,1 auf 14,3 Prozent. 